\title{
SELECCIÓN DE TRES HÍBRIDOS DE PIMIENTO (CAPSICUM ANNUM L.) PARA PUERTO LA BOCA, ECUADOR
}

\author{
AUTORES: Julio Gabriel Ortega ${ }^{1}$ \\ Eber Erazo Cajape ${ }^{2}$ \\ Raquel Vera Velázquez ${ }^{3}$ \\ Washington Narváez Campana ${ }^{4}$ \\ Carlos Castro Piguave ${ }^{5}$
}

DIRECCIÓN PARA CORRESPONDENCIA: (Julio.gabriel@unesum.edu.ec )

Fecha de recepción: 10/11/2021

Fecha de aceptación: 02/01/2022

\section{RESUMEN}

Esta investigación se realizó en un invernadero de la zona de Puerto La Boca de la Parroquia Puerto Cayo, Cantón Jipijapa, en la provincia de Manabí, Ecuador. El objetivo fue evaluar y seleccionar tres híbridos de pimiento por sus características agronómicas y de producción. El experimento se implementó en un diseño experimental de filas y columnas con 10 repeticiones, cada hilera tuvo 80 plantas y cada unidad experimental estuvo constituida por 27 plantas/tratamiento, de las cuales se eligieron cinco plantas al azar de cada una de las unidades experimentales en cada repetición para la toma de datos de las variables de respuesta. Las variables de respuesta fueron el número de frutos, peso del fruto, alto del fruto, ancho del fruto, grosor de tallo, altura de planta y daño por oídium (hongo). El hibrido que presentó el mayor rendimiento de pimiento fue el Macantro con un peso del pimiento de 143,75 g en promedio, lo que indica que este híbrido se adaptó bien a las condiciones de invernadero de Puerto la boca. Los resultados mostraron que el mejor pimiento según las características agronómicas y de preferencia por el mercado y que tuvo una buena relación entre peso de frutos, altura de planta y días a la cosecha fue el híbrido Macantro, seguido de Tandara.

PALABRAS CLAVE: Rendimiento; adaptación; agricultores; mercado; hortaliza.

\footnotetext{
1 Docente investigador, Universidad Estatal del Sur de Manabí (UNESUM), Jipijapa, Ecuador. E-mail: Julio.gabriel@unesum.edu.ec. ORCID: https://orcid.org/0000-0001-9776-9235 :

2 Técnico Empresa Pública UNESUM, Manabí, Ecuador. E-mail: erazo-eber6651@unesum.edu.ec. ORCID: https://orcid.org/0000-0002-6769-6685

${ }^{3}$ Universidad Estatal del Sur de Manabí. Docente carrera Agropecuaria, Jipijapa, Manabí, Ecuador. E-mail: veraraquel@unesum.edu.ec. ORCID: https://orcid.org/0000-0002-5071-7523.

4 Docente investigador, Universidad Estatal del Sur de Manabí (UNESUM), Jipijapa, Ecuador. E-mail: Washington.narvaez@unesum.edu.ec. ORCID: https://orcid.org/0000-0002-6674-2088

5 Docente investigador, Universidad Estatal del Sur de Manabí (UNESUM), Jipijapa, Ecuador. E-mail: Carlos.castro@unesum.edu.ec. ORCID: https://orcid.org/0000-0003-3180-2359
} 
Julio Gabriel, Eber Erazo, Raquel Vera, Washington Narváez, Carlos Castro

\title{
SELECTION OF THREE HYBRIDS OF PEPPER (CAPSICUM ANNUM L.) FOR PUERTO LA BOCA, ECUADOR
}

\begin{abstract}
This research was carried out in a greenhouse in the Puerto La Boca area of the Puerto Cayo parish, Jipijapa Canton, in the province of Manabí, Ecuador. The objective was to evaluate and select three pepper hybrids for their agronomic and production characteristics. The experiment was implemented in an experimental design of rows and columns with 10 repetitions, each row had 80 plants and each experimental unit consisted of 27 plants/treatment, of which five plants were chosen at random from each of the experimental units in each repetition for data collection of the response variables. The response variables were the number of fruits, fruit weight, fruit height, fruit width, stem thickness, plant height and damage by oidium (fungus). The hybrid that presented the highest pepper yield was the Macantro with a pepper weight of $143.75 \mathrm{~g}$ on average, which indicates that this hybrid adapted well to the greenhouse conditions of Puerto la boca. The results showed that the best pepper according to agronomic characteristics and preference for the market and that it had a good relationship between fruit weight, plant height and days to harvest was the hybrid Macantro, followed by Tandara.
\end{abstract}

KEYWORDS: yield; adaptation; farmers; market; vegetables.

\section{INTRODUCCIÓN}

La planta del pimiento fue extraída de México de las expediciones que hicieron los españoles, se dice que llegaron en el viaje que realizó Cristóbal Colon a América en el año de 1943. Sin embargo, los nativos ya conocían la planta y su fruto con el nombre de chili, pero los españoles y portugueses lo bautizaron como pimiento (Jessi, 2015).

El cultivo de pimiento ornamental es una práctica arraigada tanto en el mercado de plantas ornamentales como el mercado culinario. Los colores vivos de los pimientos y sus tamaños únicos provocan interés a los aficionados de las plantas ornamentales, y el sabor a los aficionados de pimientos picantes. En el Ecuador se estima que se siembra alrededor de 1420 ha, con una producción que bordea las 6955 toneladas y un rendimiento promedio de 4,58 t/ha (Jovicich et al., 2004).

En el Ecuador, durante mucho tiempo, se viene cultivando pimiento a campo abierto, sin contar con el interés por parte de los agricultores de tratar de mejorar el cultivo, ampliando sus alternativas y realizando su trabajo en diferentes ambientes y métodos productivos, en este caso específico bajo condiciones de invernadero, teniendo como beneficio directo la obtención de información técnica y científica sobre su producción bajo este tipo de condiciones. En el país se siembran cuatro variedades de pimientos. La variedad Quetzal es conocido como el pimiento de las 'tres puntas' por las protuberancias de su parte superior. El salvador es el más resistente a las lluvias. Estas se producen en la costa y en Loja, donde los agricultores de Santa Elena se dividen en dos grupos de productores: los que reciclan las semillas (de los pimientos que no venden) y quienes compran semillas autorizadas para la producción de este cultivo. Entre la diferencia de las semillas está el valor de la inversión. Los primeros ahorran dinero al sacar 1000 semillas de 20 pimientos y los segundos gastan USD 42 para comprar un sobre con la misma cantidad según la Asociación de Productores Hortofrutícolas de la Costa (Ashofruco). Santa Elena ocupa el

64 UNESUM-Ciencias. Publicación cuatrimestral. Vol. 6, Año 2022, No. 2 (Especial Agropecuaria) 
primer lugar con 150 hectáreas. Le siguen la Sierra Norte, Manabí y Loja. Por el saco de 70 libras el agricultor recibe USD 9,00 de los comerciantes mayoristas (Erazo y Gabriel, 2018).

La presente investigación se realizó para que exista en la zona Sur de Manabí en Puerto la Boca, donde se evaluó híbridos de pimiento de alto potencial genético, debido a que en estas zonas cultivan variedades susceptibles a enfermedades y esto ocasiona pérdidas en la producción que oscilan hasta en un 40\%, afectando al ingreso económico de los agricultores de Puerto la Boca, Manabí. Puerto La Boca de la Parroquia Puerto Cayo, Cantón Jipijapa, es una comunidad muy productiva gracias a su clima. Los habitantes de este lugar por muchos años se han dedicado a la agricultura, la pesca y una mínima parte de esta población se ha dedicado al turismo. A pesar de ser una comunidad ubicada muy cerca al mar, su agua es dulce lo cual permite cultivar en la época de verano con sistemas de riego.

Las variedades Quetzal, Tropical, Irazú y Nathalie son utilizados por los agricultores en la zona de Puerto la Boca, estas son susceptibles a enfermedades como el mildiu causado por el Oomycete Phytophthora infestans y nematodos como Meloydogyne sp. Asimismo los cultivos utilizados son susceptibles a plagas. Todos estos factores, ocasionan pérdidas que oscilan entre 30 a $60 \%$ si no se controlan adecuadamente. Por las razones mencionadas, existe la necesidad de evaluar la adaptación de nuevos híbridos, que tengan mejores características de resistencia a plagas y enfermedades, tengan un alto potencial de rendimiento y satisfagan la demanda de los consumidores.

Se debe considerar que en el mercado, el acceso a la compra de semilla certificada de alto potencial genético es limitado, por lo que se hace necesario implementar este tipo de investigaciones, que permitan identificar nuevos genotipos de pimiento de alta productividad y con buena adaptación a la zona de cultivo, para poder fomentar la siembra y cubrir la demanda insatisfecha que existe en muchos meses del año.

Por todo lo antes expuesto, el estudio tuvo como objetivo, evaluar híbridos de pimiento (Capsicum annum L.) bajo condiciones de invernadero en la zona de Puerto la Boca de la Parroquia Puerto Cayo, Cantón Jipijapa, Manabí.

\section{DESARROLLO}

La investigación fue desarrollada en un invernadero del Recinto Puerto la Boca perteneciente a la parroquia Puerto Cayo del cantón Jipijapa, que está ubicado en la latitud: $1^{\circ} 18 ' 20$ "S y longitud: $80^{\circ} 45^{\prime} 42^{\prime \prime} \mathrm{O}$, a una altitud aproximada de $53 \mathrm{msnm}$; su clima posee una temperatura de $24.8^{\circ} \mathrm{C}$; la precipitación promedio anual es de $298 \mathrm{~mm}$, concentrándose la mayor cantidad de lluvia en el mes de febrero, mientras que el mes más seco es en agosto (Gabriel et al., 2020).

\section{Tratamientos}

Los tratamientos fueron dos híbridos de pimiento y un testigo (hibrido de pimiento más sembrado en la zona).

- Tandara F1, proveniente de la empresa holandesa ENZA ZADEN (Enza zaden, 2018)

- E20L30100 F1, proveniente de la empresa ENZA ZADEN (Enza zaden, 2018)

- Macantro F1 (testigo), proveniente de la TEZIER, distribuido por Alaska S.A 


\section{Diseño experimental}

Los tratamientos de pimiento fueron distribuidos en un diseño experimental de filas y columnas con 10 repeticiones (Gabriel et al., 2021). Este diseño permitió bloquear el efecto de luz solar en el invernadero y la fertilidad en las unidades experimentales. Cada hilera tuvo un total de 80 plantas y cada unidad experimental estuvo constituida por 27 plantas/tratamiento, de las cuales se eligieron cinco plantas al azar de cada unidad experimental en cada repetición para evaluar las variables de estudio. En total se evaluaron 150 plantas. La distancia de siembra fue de $0,40 \mathrm{~m}$ entre planta y $1.20 \mathrm{~m}$ entre hileras. El número total plantas en el experimento fueron de 800 plantas.

\section{Variables de estudio}

Estas variables de estudio fueron detalladas por Erazo y Gabriel (2019).

Altura de planta (cm). - Se evaluó la altura de planta con la ayuda de un flexómetro, en 150 plantas tomadas al azar dentro de la parcela útil (cinco plantas/unidad experimental).

Diámetro de tallo (cm). Se efectuó la toma del diámetro de tallo con la ayuda de un calibrador vernier o pie de rey en 150 plantas tomadas al azar dentro de la parcela útil.

Número de frutos por planta $\left(\mathbf{N}^{\circ}\right)$. Se consideró las 150 plantas seleccionadas al azar dentro de la parcela útil.

Peso de frutos por planta (g). Este dato se tomó de los frutos cosechados de las 150 plantas seleccionadas al azar, utilizando una balanza gramera digital.

Largo de frutos (cm). El largo de frutos se midió con la ayuda de una cinta métrica, se tomó uno a uno los frutos para sacar este dato.

Ancho de frutos (cm). El ancho de frutos se tomó considerando en la parte intermedia del fruto con la ayuda de una cinta métrica.

Alto de frutos (cm). El alto del fruto se tomó con la ayuda de una cinta métrica desde la base hasta la parte más alta por tratamiento.

Volumen total de frutos (cm3). Para determinar el volumen de cada fruto se utilizó la formula recomendada por Martel Moreno (1999), que considera el largo, ancho y alto del fruto.

\section{Análisis estadísticos}

Sobre la base en el modelo definido y previo el análisis de normalidad (Gabriel et al., 2021) y homogeneidad de varianzas (Morrison, 1976) se realizó el análisis de varianza, para probar hipótesis acerca de los efectos fijos, así como las comparaciones de medias de los tratamientos mediante la prueba de tukey al $\operatorname{Pr}<0,05$ de probabilidad. El análisis de varianza también sirvió para estimar los componentes de varianza para los efectos aleatorios. Los análisis indicados se realizaron mediante el Proc GLM del SAS (Gabriel et al., 2021).

Se realizó el análisis de correlación entre las variables de estudio utilizando el coeficiente de Pearson sugerido por Gabriel et al. (2021).

\section{Análisis beneficio/costo (B/C)}


Se determinó el benéfico/costo de cada tratamiento aplicado. Este análisis permitió definir la rentabilidad o no de los tratamientos. Si el resultado de la relación B/C fue mayor a 1 será rentable (Boardman et al., 2018).

\section{Resultados}

Análisis de normalidad y de homogeneidad de varianzas

Los análisis de normalidad y análisis de homogeneidad de los datos de las variables como el número de frutos promedio (NFP), peso del fruto (PFr), largo del fruto (LFr), ancho del fruto (AFr), diámetro de tallo (DT), altura de la planta (AP) y severidad a oídium (SEVO), no fueron significativos a las pruebas de Kosmorolov-Smirnov y Chi-cuadrada al $\operatorname{Pr}<0,05$ de probabilidad, respectivamente. Ambos análisis sugirieron proceder con los análisis de varianza y la comparación de medias.

\section{Análisis de varianza}

El análisis de varianza de las variables NFP, PFr, LFr, AFr, DT, AP y SEVO, mostraron que los coeficientes de variación (CV), están dentro de los rangos permitidos para este tipo de investigación (C.V. de 10.07\% a 27,69) (Tabla 1).

Se observó diferencias altamente significativas para los tratamientos al $\operatorname{Pr}<0,01$ de probabilidad para todas las variables evaluadas. Esto está indicando que al menos uno de los tratamientos fue diferente (Tabla 1).

Tabla 1. Análisis de varianza para variables evaluadas, número de frutos promedio, peso de fruto, volumen de fruto, diámetro de tallo, altura de planta y severidad al oídium.

\begin{tabular}{|c|c|c|c|c|c|c|c|}
\hline \multirow{2}{*}{$\mathbf{F V}$} & \multirow{2}{*}{ Gl } & \multicolumn{6}{|c|}{ Cuadrados medios } \\
\hline & & NFP & PFr & VFr & DT & AP & SEVO \\
\hline Total & 149 & & & & & & \\
\hline Tratamiento & 2 & $1074,42 * *$ & $51666,33^{* *}$ & $431293995,6 * *$ & $0,26 * *$ & $4512,28 * *$ & $7421,04^{* *}$ \\
\hline Hileras & 9 & $12,18^{*}$ & $5,51 \mathrm{~ns}$ & $1,9 \mathrm{~ns}$ & $0,00 \mathrm{~ns}$ & $22,42 n s$ & $0,00 \mathrm{~ns}$ \\
\hline Columnas & 2 & $4,00 \mathrm{~ns}$ & $6,17 \mathrm{~ns}$ & $1,5 \mathrm{~ns}$ & $0,00 \mathrm{~ns}$ & $25,14 \mathrm{~ns}$ & $0,00 \mathrm{~ns}$ \\
\hline Error & 136 & 5,87 & 314,25 & 25578175 & 0,03 & 212,35 & 16,35 \\
\hline CV & & 10,07 & 18,17 & 25,53 & 15,46 & 16,31 & 27,69 \\
\hline
\end{tabular}

**: Diferencias altamente significativas al $\operatorname{Pr}<0,01$ de probabilidad. ns: No significativo, CV: Porcentaje de Coeficiente de Variación. NPF: número de frutos promedio, PFr: peso de fruto, VFr: volumen de fruto, DT: diámetro de tallo, AP: altura de planta y SEVO: severidad al oídium.

El análisis de medias realizado mediante la prueba de Tukey al $\operatorname{Pr}<0,05$ de probabilidad para las variables NFP, PFr, VFr, DT, AP y SEVO, mostró diferencias significativas en todos los casos (Tabla 2). 
Tabla 2. Análisis de medias mediante la prueba de tukey al $\operatorname{Pr}<0,05$ de probabilidad para las variables evaluadas.

\begin{tabular}{lcccccc}
\hline Cultivar & NFP & PF & VF & DT & AP & SEVO \\
\hline E20L.30100 & $29,26 \mathrm{a}$ & $68,42 \mathrm{c}$ & $17584 \mathrm{c}$ & $1,18 \mathrm{~b}$ & $89,96 \mathrm{~b}$ & $3,00 \mathrm{c}$ \\
Tandara & $22,76 \mathrm{~b}$ & $92,00 \mathrm{~b}$ & $18681 \mathrm{~b}$ & $1,28 \mathrm{a}$ & $98,96 \mathrm{a}$ & $27,40 \mathrm{a}$ \\
Macantro & $20,16 \mathrm{c}$ & $132,25 \mathrm{a}$ & $23156 \mathrm{a}$ & $1,14 \mathrm{~b}$ & $80,00 \mathrm{c}$ & $13,40 \mathrm{~b}$ \\
DSH & 1,14 & 8,40 & 2396,9 & 6,90 & 6,90 & 1,92 \\
\hline
\end{tabular}

Medias con la misma letra no son significativamente diferentes al $\operatorname{Pr}<0,05$ de probabilidad. NPF: número de frutos promedio, PFr: peso de fruto, VFr: volumen de fruto, DT: diámetro de tallo, AP: altura de planta y SEVO: severidad al oídium.

El NPF fue significativo al $\operatorname{Pr}<0,05$ de probabilidad, el hibrido E20L30100, mostró ser el mejor con hasta 29 frutos por planta, seguido de Tandara con 23 frutos y Macantro con 20 frutos (Tabla 2).

En referencia al PFr también fue significativo al $\operatorname{Pr}<0,05$ de probabilidad, mostrando que el mejor cultivar fue Macantro con un peso promedio de fruto de $132 \mathrm{~g}$, seguido de Tandara con $23 \mathrm{~g}$ y E20L30100 con 29 g (Tabla 2).

El VFr fue significativo al $\operatorname{Pr}<0,05$ de probabilidad, observándose un mayor VFr en el híbrido Macantro con 23156 cm3, seguido de Tandara con 186821 cm3 y E20L30100 con 17584 cm3 (Tabla 2).

Para el DT se fue significativamente al $\operatorname{Pr}<0,05$ de probabilidad, siendo el híbridos Tandara mejor con 1,28 cm, seguido del híbrido E20L30100 con 1,18 cm de DT y Macantro con 1,14 cm (Tabla 4).

En referencia a la severidad del oídium del pimiento causado por Leveillula taurica, que corresponde con la forma asexual de Oidiopsis spp, se encontró que el híbrido E20L30100 tuvo un 3\% de infección, respecto de los híbridos Macantro y Tandara, que tuvieron una severidad del $13 \%$ y $27 \%$, respectivamente (Tabla 2).

\section{Análisis de correlación}

Por otra parte, el análisis de correlación mediante el coeficiente de Pearson (Tabla 3), mostró una moderada correlación negativa y altamente significativa al $\operatorname{Pr}<0,01$ de probabilidad entre NFP con PF (-0.63), NPF con SEVO (-0.48). Así mismo, se observó una moderada correlación positiva y altamente significativa al $\operatorname{Pr}<0,01$ de probabilidad entre PF con VF $(0,49)$.

Tabla 3. Análisis de correlación para las variables evaluadas.

\begin{tabular}{lllllll}
\hline & NFP & PFr & VFr & DT & AP & SEVO \\
\hline NFP & 1,00 & $-0,63^{* *}$ & $-0,31^{* *}$ & $0,05 \mathrm{~ns}$ & $0,12 \mathrm{~ns}$ & $-0,48^{* *}$ \\
PFr & & 1,00 & $0,49^{* *}$ & $-0,14 \mathrm{~ns}$ & $-0,23^{* *}$ & $0,23^{* *}$ \\
VFr & & & 1,00 & $-0,09 \mathrm{~ns}$ & $-0,12 \mathrm{~ns}$ & $0,10 \mathrm{~ns}$ \\
AP & & & & 1,00 & $-0,13 \mathrm{~ns}$ & $0,22^{* *}$ \\
\hline
\end{tabular}

68 UNESUM-Ciencias. Publicación cuatrimestral. Vol. 6, Año 2022, No. 2 (Especial Agropecuaria) 
**: Diferencias altamente significativas al $\operatorname{Pr}<0,01$ de probabilidad, ns: No significativo, NPF: número de frutos promedio, PFr: peso de fruto, VFr: volumen de fruto, DT: diámetro de tallo, AP: altura de planta y SEVO: severidad al oídium.

\section{Análisis de beneficio/costo (B/C)}

El análisis de B/C se realizó para un invernadero de $1000 \mathrm{~m}^{2}$ para cada una de los híbridos. El análisis de Beneficio Neto, mostró que en general todos los híbridos de pimiento tuvieron un Beneficio Neto que oscilan entre USD 999,86 (I10E30100) a USD 3868,67 (Macantro). El análisis de rentabilidad, se realizó comparando en Beneficio Neto y el Costo de Producción. Esta relación tuvo que ser mayor a 1, si la tecnología era rentable. En la presente investigación se observó que los híbridos Tandara y Macantro fueron mayores a 1 (3,09 a 6,57 respectivamente).

Se hizo un análisis de comercialización para determinar las diferencias de la venta a supermercados y mercado local (abasto). Este análisis mostró que la ganancia de venta a supermercados fue superior en más del 30\% respecto del mercado local (abasto).

\section{Discusión}

Si bien en la presente investigación se observó que el híbrido más utilizado actualmente es el Macantro, sin embargo, no debemos olvidar que es necesario evaluar y seleccionar nuevos híbridos, para que en un momento sea reemplazada, por otras de mejor adaptación y rendimiento. El rendimiento como es bien conocido está influenciado por factores ambientales tal como lo sugiere Almanza (2016), así el híbrido tipo lamuyo Tandara, desarrollado por la empresa Enza Zaden (2018), es de alta productividad, muy rústica y se adapta bien a diferentes regiones. Su peso promedio de fruto es de 200 g, resistente a los virus PVY (PVY: 0), Pepper Motle Virus (Pepmov) y Tabomovirus (Tm: 0).

Bien podría este híbrido, ser cultivado en la zona de Puerto la Boca, como alternativa para los productores. Se observó que, además cuenta con un buen nivel de resistencia al oídium, una enfermedad que cada vez va tomando mayor importancia. Se notó que el híbrido Macantro fue más susceptible que los híbridos introducidos. Esto denota la importancia de probar nuevos híbridos. Así mismo se observó que el híbrido E20L30100, fue aún más resistente que Macantro y Tandara, aunque tuvo menor producción. Estos resultados son novedosos, ya que no existen reportes en otras partes de la resistencia a oídium de los materiales utilizados en esta investigación.

El diámetro del tallo de la planta orienta a que, entre mayor sea el valor para esta variable, mayor es la capacidad para soportar el peso de órganos principales como ramas, flores y frutos, y a su vez disminuye el riesgo de que el tallo se quiebre por un exceso de peso de la parte aérea de la planta. En pimiento se tienen informes de que el diámetro del tallo, al utilizar una densidad de siembra entre 2,5 - 6,0 plantas/ $\mathrm{m}^{2}$, puede variar entre 14,0 y 27,3 mm (Grijalva et al., 2008; Moreno et al., 2011). En otro trabajo de investigación, encontraron resultados de diámetro de tallo por debajo de lo informado en la literatura, con un valor mínimo de 12,55 mm (Elizondo y Monge, 2017). En nuestra investigación, hubo diámetros de tallo de 11,4 mm 11,8 mm, que son menores a los reportados, esto posiblemente debido a que aún es necesario investigar sobre la mejor fertilización de los híbridos para el engrose del tallo. 
En referencia a la altura de la planta se puede decir que varió significativamente entre los genotipos evaluado, tal como fue observado por Elizondo y Monge (2017), quienes mencionan que, para el caso de genotipos de pimiento de forma cuadrada, generalmente se presenta una mayor altura al aumentar el número de ramas. Enfatizan que la altura de la planta es una característica varietal que se ve influenciada por la interacción genotipo-ambiente; por lo tanto, hay factores como la temperatura, humedad, manejo del cultivo y calidad de la semilla que pueden influir en las diferencias entre genotipos (Eliozondo y Monge, 2017). Una de las características principales que debe presentar el cultivo de pimiento es un buen desarrollo vegetativo, con adecuada cobertura de hojas, ya que esto reduce el riesgo de quemaduras del fruto por exposición solar, pues generalmente las plantas más pequeñas y compactas presentan una mejor cobertura de los frutos (Monge Pérez, 2017).

En pimiento existe reportes de que la altura de la planta, al utilizar una densidad de siembra entre 1,56 - 6,0 plantas $/ \mathrm{m}^{2}$ puede variar entre 0,49 y 2,24 m (Jovicich et al., 2004; Moreno et al., 2011; Paunero, 2008; Melgar et al., 2010). Los datos obtenidos en este trabajo coinciden con lo informado en la literatura. En algunas ocasiones la altura de la planta es mayor conforme aumenta la densidad de siembra (Jovicich et al., 2004; Seifi et al., 2012), pero en otras ocasiones se ha encontrado el resultado contrario (Hossein et al., 2012), o no se han encontrado diferencias significativas entre distintas densidades de siembra (Melgar et al., 2010, Elizondo y Monge, 2017). En nuestra investigación el promedio de altura estuvo entre 0,80 m (Marcantro) a 0,98 m (Tandara).

Se observó, que el mejor pimiento según sus características agronómicas y de altura de planta y diámetro de tallo fue el Tandara, al respecto Bosch (2013), menciona que el pimiento puede cultivarse durante todo el año, pero hay que tener en cuenta que se requieran temperaturas moderadas o cálidas, esto será lo que defina la época de siembra y cosecha es por esta razón que generalmente el horticultor busca forzar estos cultivos haciéndolos fuera de época en busca de precios más favorables, es por ello que se adoptan nuevas tecnologías, como cultivar en invernadero, el uso de mallas plásticas que intercepten más del 50 \% la luz del sol, y mejorar el sistema de riego.

El hibrido que presentó el mayor rendimiento de pimiento en invernadero fue el Macantro con un peso de 143,75 g en promedio, lo que indica que este hibrido se adaptó bien a las condiciones de invernadero de Puerto la Boca. Enza Zaden (2018) se reportó que el híbrido Tandara puede producir un peso promedio de fruto de $200 \mathrm{~g}$.

Se debe destacar que, en Ecuador los consumidores prefieren las variedades tipo lamuyo que son alargados y cuadrados con carne gruesa, parecidos a un ají y no están acostumbrados a consumir los pimentones tipo california con tres o cuatro cascos bien marcados.

Por otra parte, Arévalo (2015), indica que es importante identificar el hábito de crecimiento para el tipo de pimiento que se quiere sembrar, debido a que las plantas difieren unas de otras en su poder de absorción, aquellas plantas diferentes cultivadas en un mismo suelo pueden poseer una alimentación mineral diferente desde un punto de vista cualitativo como cuantitativo. Incluso cultivares diferentes de una misma especie vegetal no procede de la misma forma. En esta investigación, si bien no analizamos el hábito de crecimiento, se vio la importancia de hacer un buen tutoraje y una poda apropiada, para prevenir el ataque de enfermedades principalmente. 
Según (Elizondo y Monge, 2017) el crecimiento y desarrollo del cultivo puede variar según los genotipos y el potencial de rendimiento a mayor producción en un ambiente protegido donde se controla con mayor facilidad el turado, poda, riego y manejo de fertilizantes.

El análisis de correlación realizado en la investigación mostro una relación negativa altamente significativa entre el número promedio de frutos y el peso de fruto, esto está denotando que a mayor número de frutos va en detrimento del peso de los frutos. Así mismo fue notorio observar que también hubo una relación negativa y altamente significativa entre el número promedio de frutos y la Severidad del oídium, esto estaría indicando que a la severidad del oídium es más notoria cuando hay menos frutos. También se observó una alta correlación moderada positiva y significativa ente el peso de frutos y el volumen de frutos, lo que estaría dando a entender que los frutos más grandes son los más pesados. Esto posiblemente al tipo de espesor de la pared del fruto (Elizondo y Monge, 2017).

El análisis de costos de cada híbrido evaluado mostró que la mejor alternativa económica por tener una relación beneficio/costo superior a la media, fueron los híbridos de pimiento Macantro y Tandara, con 3,09 a 6,57, respectivamente de relación beneficio/costo

\section{CONCLUSIONES}

Los mejores híbridos fueron Macantro y Tandara. Este último híbrido mostró tolerancia a oídium, que es una enfermedad que cada vez se vuelve más importante.

Ambos híbridos fueron rentables para su venta a supermercados y mercados de abasto, siendo buenas alternativas tecnológicas para su cultivo bajo invernadero en Puerto la Boca.

\section{AGRADECIMIENTOS}

Los autores agradecen el apoyo financiero y las facilidades proporcionadas por la Universidad Estatal del Sur de Manabí (Grant PROG-003-PROY-001-DIP-2017 a JGO de la UNESUM). También agradecemos a los agricultores de Puerto La Boca y a todos los estudiantes involucrados en esta investigación.

\section{REFERENCIAS BIBLIOGRÁFICAS}

Almanza, P., Arévalo, A., Cely, G., Pinzón, E., \& Serrano, P. (2016). Caracterización del crecimiento del fruto de tomate (Solanum lycopersicum L.) híbrido 'Ichiban' cultivado bajo cubierta. Agron. Colombia, 34,155-162. https://doi.org/10.15446/agron.colomb.v34n2.57193

Arévalo, G. (2015) determinación de la eficiencia de manejo de nutrientes y agua en tres variedades de pimiento de color (Capsicum annum) bajo hidroponía en condiciones de invernadero en mulacagua, departamento de Comayagua. Honduras. Tesis, Universidad de Almeria, España. https://dialnet.unirioja.es/servlet/tesis?codigo=111599

Boardman, N. E., Greenberg, D., Vining, A. y Weimer, D. (2018). Cost-benefit Analysis: Concepts and Practice (5st edition). Cambridge University Prees, UK. http://students.aiu.edu/submissions/profiles/resources/onlineBook/E5V5H3_Costbenefit\%20analysis\%20_\%202018.pdf

Bosch, M.B. (2013). Manual Práctico de huerto ecológico. Fertilidad de la Tierra. España.

Elizondo, E. y Monge, J.E. (2017). Evaluación de rendimiento y calidad de 15 genotipos de pimiento (Capsicum annuum L.) cultivados bajo invernadero en Costa Rica. Revista Tecnología en Marcha, 30(4), 3-14. https://doi.org/10.18845/tm.v30i4.3407

(C) Universidad Estatal del Sur de Manabí. Jipijapa, Ecuador. 
Julio Gabriel, Eber Erazo, Raquel Vera, Washington Narváez, Carlos Castro

Enza Zaden. (2018). Enza Zaden Baikonur F1 E15B.50206. https://smadshop.md/selskoe-hozyajstvo/semenatomata-rannespelogo-enzazaden-baikonur-f1-e15b.50206-250-semyan.html

Erazo, E. y Gabriel, J. (2018). Evaluación y selección de cultivares híbridos de pimiento (Capsicum annum L.) bajo invernadero en la zona de Puerto la Boca Manabí. Tesis, Universidad Estatal del Sur de Manabí, Jipijapa, Ecuador. http://repositorio.unesum.edu.ec/

Gabriel, J, Delvalle, J., Padilla, J., Pincay, N., Ayón, F., Narváez, W. y González, A. (2020). Innovaciones en la matriz productiva hortícola para reducir el efecto del cambio climático en Puerto la Boca, Jipijapa, Ecuador. Journal of the Selva Andina Research Society, 11(1), 2-17. http://dx.doi.org/10.36610/j.jsars.2020.110100002

Gabriel, J., Valverde, A., Indacochea, B., Castro, C., Vera, M., Alcívar, J., Vera, R., (2021). Diseños experimentales: Teoría y práctica para experimentos agropecuarios. Segunda edición, Editorial Grupo Compás. Universidad

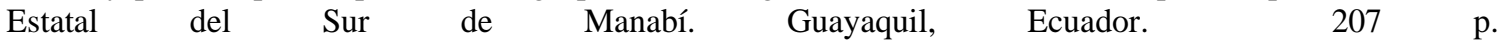
http://142.93.18.15:8080/jspui/handle/123456789/625

Grijalva-Contreras, R.L., Robles-Contreras, F., Macías-Duarte, R., Santillano-Cázares, J., y Núñez-Ramírez, F. (2016). Nitrógeno en trigo y su efecto en el rendimiento y en la concentración de nitratos y potasio en el extracto celular de tallo (ECT). Acta Universitaria, 26 (5), 48-54. http://dx.doi.org/10.15174/au.2016.963

Hossein, M., Aroiee, H., Ameri, A. and Fatemi, H. (2012). Effect of plant density and nitrogen fertilizer on growth, yield and fruit quality of sweet pepper (Capsicum annum L.). Academic Journal,7(6), 859-866. https://doi.org/10.5897/AJAR10.505

Jessi Bloom, D. B. (2015). Permacultura Práctica: para tu terreno, y todo el planeta. La Fertilidad de la Tierra Ediciones, España.

Jovicich, E., Cantliffe, J.D., and Stoffella, P.J. (2004). Fruit Yield and Quality of Greenhouse-grownBell Pepper as Influenced by Density, Container, and Trellis System. HortTechnology, 14(4), 507-513. https://doi.org/10.21273/HORTTECH.14.4.0507

Melgar, R., Clemente, R., Pérez, M., Esaú, del C.; Sánchez, F., Rodríguez Pérez, J. y Peña Lomelí, A. (2010). Variedades de pimiento morrón manejadas con despunte temprano en dos densidades de población. Revista Chapingo Serie Horticultura, 16(3), 223-229. https://www.redalyc.org/pdf/609/60919865010.pdf

Monge Pérez, J. (2017). Efecto de la poda y la densidad de siembra sobre el rendimiento y calidad del pimiento cuadrado (Capsicum annuum L.) cultivado bajo invernadero en Costa Rica. Tecnología en Marcha, 29(2), 125-136. https://doi.org/10.18845/tm.v29i2.2696

Moreno, Claudia E.; Barragán, Felipe; Pineda, Eduardo y Pavón, Numa P. (2011). Reanálisis de la diversidad alfa: alternativas para interpretar y comparar información sobre comunidades ecológicas. Revista Mexicana de Biodiversidad, 82: 1249-1261. http://www.scielo.org.mx/scielo .

Morrison, D.F. (1976). Species of the Genus Uniomerus Multivariate Statistical Methods. McGraw-Hill Company, New York, 214 p. https://www.landscapepartnership.org/projects/trb/resources/

Paunero, I.E. (2008). Prevención de accidentes en la cosecha de frutales. San Pedro: Ediciones INTA, 1-6. https://inta.gob.ar/sites/default/files/script-tmp-ip_1002.pdf

Seifi, S., Nemati, S.H., Shoor, M. \& Abedi, B. (2012). The effect of plant density and shoot pruning on growth and yield of two greenhouse bell pepper cultivars. Journal of Science and Technology of Greenhouse Culture, 3(11), 77-83. http://ejgcst.iut.ac.ir/browse.php?a_...

72 UNESUM-Ciencias. Publicación cuatrimestral. Vol. 6, Año 2022, No. 2 (Especial Agropecuaria) 